\title{
Electron Bernstein Wave Experiments at the WEGA Stellarator
}

\author{
H.P. Laqua ${ }^{\mathrm{a}}$, S. Marsen ${ }^{\mathrm{a}}$, M. Otte ${ }^{\mathrm{a}}$, Y. Podoba ${ }^{\mathrm{a}}$, J. Preinhealter ${ }^{\mathrm{b}}, \mathrm{T}$. \\ Stange $^{\mathrm{a}}$, J. Urban ${ }^{\mathrm{b}}$, D. Zhang ${ }^{\mathrm{a}}$ \\ ${ }^{a}$ Max-Planck-Institut für Plasmaphysik, EURATOM Ass. D-17491 Greifswald, Germany \\ ${ }^{b}$ Institute of Plasma Physics, EURATOM/IPP.CR Ass., 18200 Prague, Czech Republic
}

\begin{abstract}
Fundamental investigations on EBW excitation, propagation and absorption are presented. In particular the OXB mode conversion and EBW current drive with $2.45 \mathrm{GHz}$ EBWs are investigated and compared with modeling. High density operation with $28 \mathrm{GHz}$ EBWs could be achieved at $0.5 \mathrm{~T}$ ( $2^{\text {nd }}$ harmonic). This regime is characterized by a additional strongly suprathermal electron distribution with energies $>10 \mathrm{keV}$. These electrons were detected by measurement of the EBW emission and by X-ray detection.
\end{abstract}

Keywords: ECRH, ECCD, Bernstein waves, stellarator, supra thermal electrons

PACS: 52.35.Hr, 52.50.Sw, 52.55.Wq

\section{INTRODUCTION}

High density and high beta operation in fusion plasmas could not be sustained by electron cyclotron resonance heating (ECRH) yet, since the total reflection at the cutoff limit prohibits the wave propagation into the dense plasma core. However, the super-dense operation in stellarators and the high beta regime in spherical tokamaks, which are alternative reactor concepts, necessitate over-dense plasma heating. In this sense ECRH with electron Bernstein waves (EBWs) is a promising candidate. It can deposit its power into a small volume, to achieve a high power per volume ratio and is able to drive a local toroidal current efficiently, which is needed to generate rotational transform and to stabilize MHD modes in tokamaks. EBWs are electrostatic plasma waves and have to be generated by the $\mathrm{OXB}$ or XB mode conversion process at the plasma edge [1]. Their propagation is highly sensitive to the magnetic field configuration. Therefore, precise EBW ray-tracing calculations are necessary in order to control the power deposition and the EBW driven current. In this paper an overview of experimental results with $2.45 \mathrm{GHz}$ and $28 \mathrm{GHz}$ EBW heating will be given.

\section{EXPERIMENTAL SET-UP}

WEGA is a classical five period and $l=2$ stellarator with a major radius of $0.72 \mathrm{~m}$ and an aspect ratio of 7 [9]. It is equipped with three independent coil systems. The toroidal field coils provide the toroidal magnetic field, the two oppositely charged 
helical field coils generate the rotational transform and thus the closed flux surfaces and the vertical field coils generate a vertical magnetic field, which can be used for radial plasma position control and shear variation.

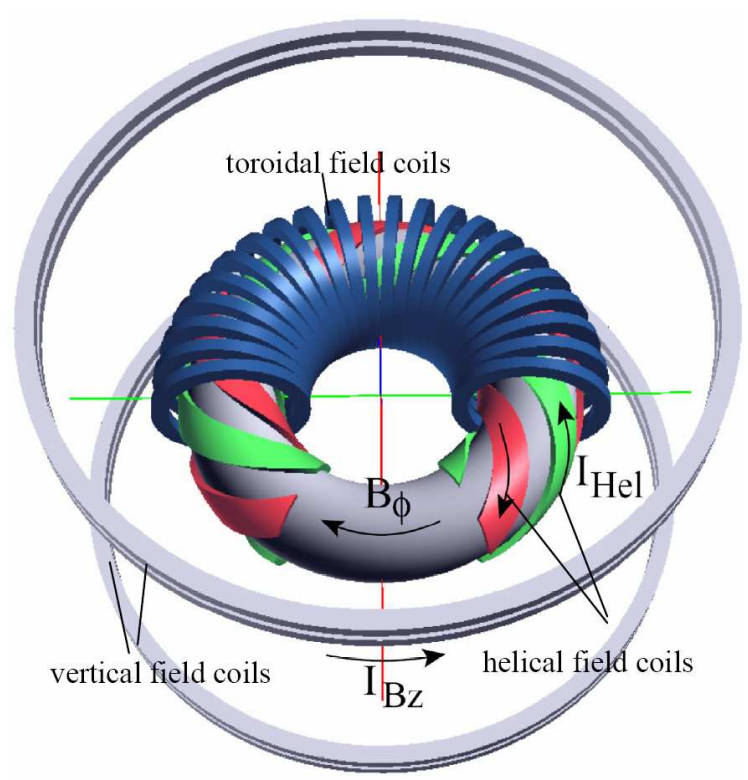

FIGURE 1. Magnetic field coils of WEGA

In addition WEGA is equipped with an iron $\mathrm{OH}$-transformer with a capability of $440 \mathrm{mVs}$. The discharge length at $0.5 \mathrm{~T}$ is typically up to $20 \mathrm{~s}$, which allows operating the plasma in a stationary state. The heating system of WEGA consists of two 2.45 $\mathrm{GHz}$ magnetrons with a total power of $26 \mathrm{~kW}$. The power is launched by two monomode transmission lines with a TE11 cylindrical antenna with a double cut at the antenna front [2] providing a k-spectrum which is optimal for OXB-conversion.

The $28 \mathrm{GHz}$ system consists of a $10 \mathrm{~kW}$ cw gyrotron with a transmission line, which is a combination of a waveguide system for mode conversion from the TE02 gyrotron mode into an elliptically polarised HE11 mode and a quasi-optical antenna system for proper beam launch into the plasma. In total only $10 \mathrm{~kW} / 0.15 \mathrm{~m}^{3}$ of ECRH power density is available, which necessitates a highly efficient OXB-mode conversion. Therefore, the $28 \mathrm{GHz}$ power was launched with a pure O2-mode elliptical polarisation under an optimal angle of $55^{\circ}$ for OXB-conversion. The beam was focussed at the plasma edge at a toroidal position with the maximum vertical plasma elongation. Here, the smallest density scale length $L_{\mathrm{n}}$ was expected. The broadness of the $N_{\|}$spectrum is reciprocal to the focus diameter. On the other hand large poloidal focus size would prohibit central power deposition. Therefore, as a compromise, a toroidally elongated focus of FWHM $4.1 \mathrm{~cm}$ in toroidal direction and $2.3 \mathrm{~cm}$ in poloidal direction was chosen. In addition, this toroidal position of symmetry features an unique magnetic configuration, where the $N_{\|}$component of the EBW remains small during the propagation. This behaviour is similar to equatorial launch in a tokamak. Here, only slightly Doppler shifted absorption and no current drive is expected. 3D EBW ray tracing calculations predict most central power deposition for a magnetic field of $0.48 \mathrm{~T}$ at the axis. The ray trajectories are shown in Fig. 2. It should be 
noticed, that this calculation is not self consistent. It is assumed that the density and temperature profiles remain unchanged, even though the deposition is changed. The operation at $0.5 \mathrm{~T}$ is also supported by a $26 \mathrm{~kW} 2.45 \mathrm{GHz} \mathrm{cw}$ magnetron system with a double-slot antenna, which provides a large $N_{\|}$to excite resistively absorbed R-waves in the plasma.
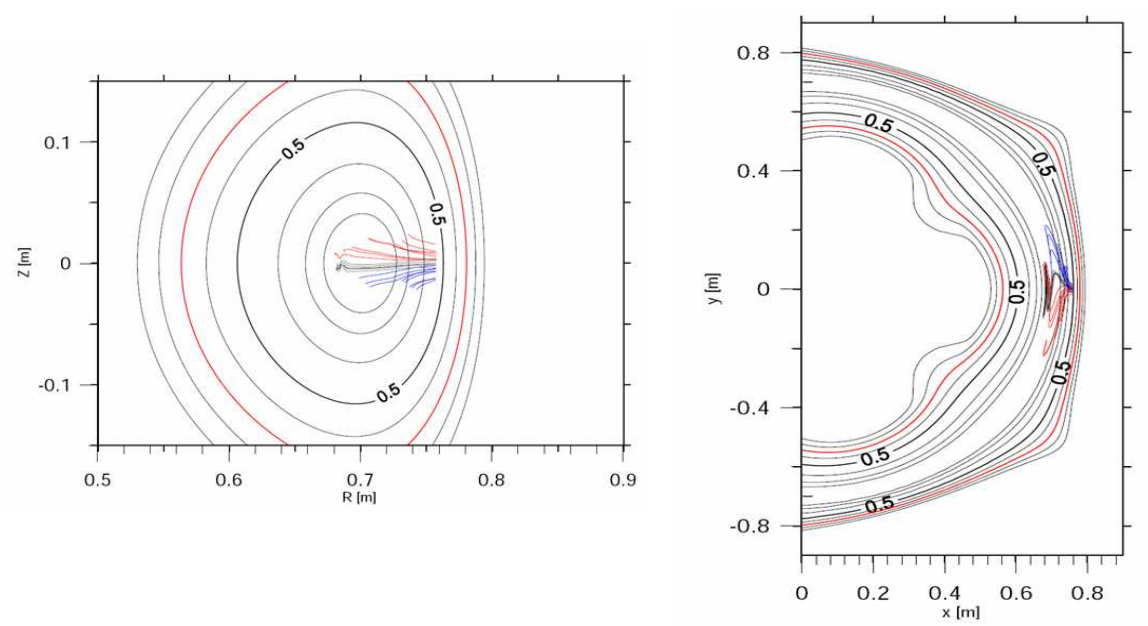

FIGURE 2. EBW ray trajectories in the projection at the poloidal (left) and toroidal (right) plasma cross section

WEGA is equipped with several diagnostics. The line averaged density is measured by a single line $80 \mathrm{GHz}$ interferometer. The radiation profile is measured by a 12 channel bolometer camera. Very useful is the so called "sniffer" probe, which measures the $28 \mathrm{GHz}$ ECRH-stray radiation level in order to estimate the absorbed ECRH power. The probe is located at a toroidal position nearly opposite to the ECRH launch antenna. Unfortunately the in-vessel diamagnetic loop was damaged by rf and was not available for the experiments. The X-ray emission can be detected by a pulse height analyser in an energy range of $0.5-15 \mathrm{keV}$. Recently a 12 channel radiometer (22.8-39.6GHz) has been installed. The antenna was viewing obliquely with an angle of $55^{\circ}$ with respect to the magnetic field vector, optimal for EBW emission (EBE) measurement by BXO conversion. The rectangular shaped horn could only detect linear polarisation, which was parallel to the magnetic field vector. Thus only a part of the elliptically polarized O-wave was detected. In addition, a second horn was installed at the high field side with a viewing angle perpendicular to the magnetic field vector for comparative measurements. Furthermore, the complete spectrum can be detected with a spectrum analyser with a sweep time of typically $300 \mathrm{~ms}$ during steady state plasma operation. The system was absolutely calibrated by the "hot-cold" method at room and liquid nitrogen temperature.

\section{HEATING AND CURRENT DRIVE WITH 2.45 GHZ EBWS}

EBWs are electrostatic waves. Therefore, they have to be exited by mode conversion from electromagnetic waves. The most favourable scheme is the two step 
conversion from an ordinary wave (O-wave) into an extraordinary wave (X-wave) and finally into a Bernstein wave. This so called OXB-mode conversion could be investigated in detail using the $2.45 \mathrm{GHz}$ heating system [2]. Here, the cut-off density is very low, therefore as a consequence the mode conversion region was shifted to the scrape-off plasma outside the separatrix, where the normalised density gradient length $\mathrm{k}_{0} \mathrm{~L}_{\mathrm{n}} \approx 5$ was favourable for OXB-mode conversion. In addition, the long wavelength provided an unique opportunity to investigate the mode conversion process in detail using radially resolved measurements of the phase and amplitude of the waves involved.
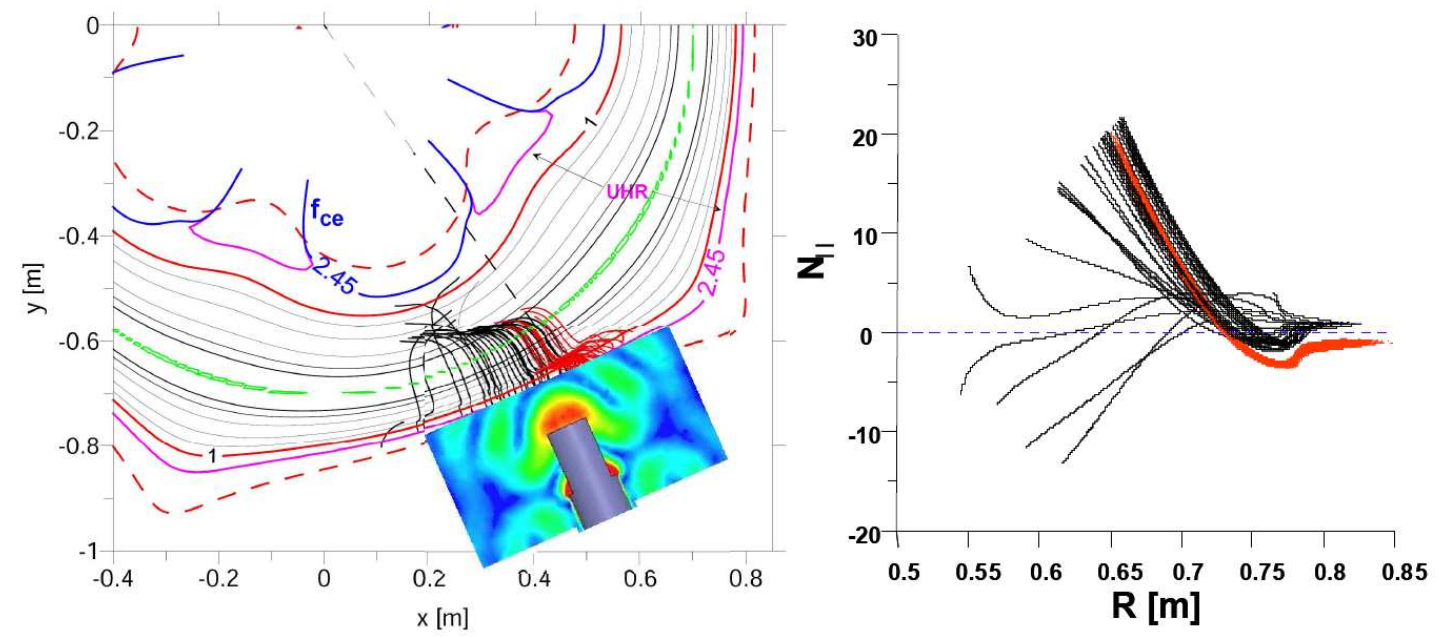

FIGURE 3. left) Ray trajectories of $2.45 \mathrm{GHz}$ EBWs in equatorial projection. right) Development of $N_{\text {II }}$ during propagation until absorption for the individual EBW rays calculated by the 3D ray-tracing code.

A two-dimensional finite-difference time-domain full wave code was developed to compare calculated and measured microwave propagation and mode conversion results. The probe measurements clearly show the transition of the O-wave into the $\mathrm{X}$ wave in the mode conversion region. The EBW heating process itself was investigated by the modulation $(12.5 \mathrm{kHz})$ of a part of the microwave power and the observation of the concomitant oscillations in the electron temperature and density. Even though the vacuum wavelength for $2.45 \mathrm{GHz}$ is of the same order as the plasma dimensions, the wavelength of the EBWs is below $0.1 \mathrm{~mm}$. Therefore, their propagation could be calculated by a 3D ray-tracing code, which also took the antenna coupling efficiency into account (see Fig. 3). The EBW rays are shown in the equatorial and poloidal projection. The code calculates the OXB conversion efficiency of the individual ray at the position, where it is generated. Even though the starting $N_{\|}$spectrum is determined by the $N_{\text {II }}$ spectrum of the emitting antenna, $N_{\text {II }}$ increases to values up to 20 when the rays propagate through the plasma. The calculation could reproduce the strongly Doppler shifted absorption, which was found in the experiments (see Fig. 4). Even though the calculated power deposition is more central the optimum is at $0.65 \cdot B_{c}$ as in the experiment. $B_{c}$ is the resonant central magnetic field for $2.45 \mathrm{GHz}(87 \mathrm{mT})$. 

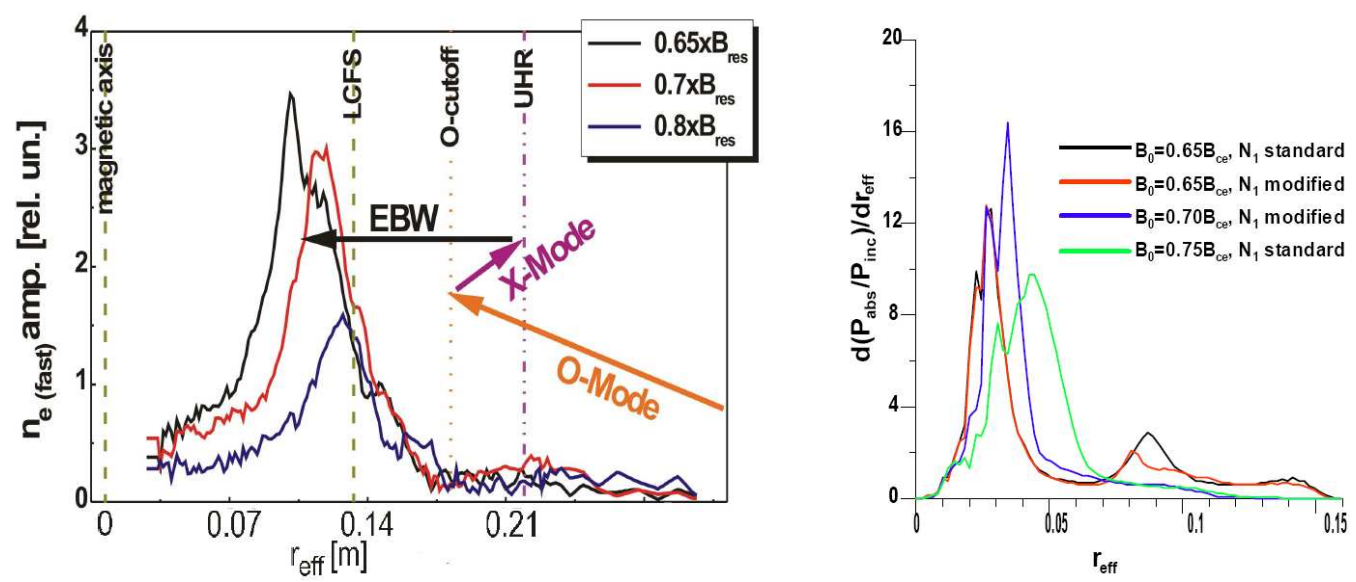

FIGURE 2. Power deposition profiles for different magnetic field strength, achieved from probe measurements (left) and ray-tracing calculations (right). In addition in the sensitivity of different profiles on the supra-thermal electrons with a temperature of $150 \mathrm{eV}$ was tested.

The change of $N_{\text {II }}$ for the EBWs is mainly affected by the magnetic field, which leads to a propagation of nearly all rays into one direction independently whether they have been emitted in co- or counter direction with respect to the magnetic field vector. The asymmetrical development of the $N_{\text {II }}$ component generates a toroidal current which is driven by EBWs. This current could be measured by an external Rogowski coil and the short-circuited primary windings of the Ohmic transformer coil. A high precision was achieved by making use of the long discharge length, which is possible at the WEGA stellarator. In typically $30 \mathrm{~s}$ the $6 \mathrm{~kW}$ magnetron power was modulated with $100 \%$ amplitude with frequencies between $27 \mathrm{~Hz}$ and $90 \mathrm{~Hz}$. A second magnetron was sustaining the plasma with additional $6 \mathrm{~kW} \mathrm{cw}$. Since the plasma was stationary for more than $25 \mathrm{~s}$, an average over all modulation cycles gives a precise measure of the driven current. The highest current was achieved at iota $=0.2$ with $90 \mathrm{~A}$ for $6 \mathrm{~kW}$ modulation and $6 \mathrm{~kW}$ constant power. The modelling of the EBW driven current in WEGA turned out to be difficult. Firstly, the profiles of density and temperature are known from probe measurement only, which give some impreciseness due to the plasma perturbation by the probe itself. Secondly, probe measurements indicate that in addition to the thermal electron component of $10-20 \mathrm{eV}$ a suprathermal population of approximately $10 \%$ in density with an energy of 150-300 eV exists. Those electrons are probably the main source of the driven current. Even more the EBW absorption takes place at the Doppler down-shifted first harmonic resonance as well as at the Doppler up-shifted second harmonic resonance. Both processes generate counter-acting currents. In addition elastic and inelastic collisions with the ions and neutrals should be taken into account, which reduces the current drive efficiency. However, only little influence of the neutral gas pressure on the CDefficiency was found in the experiments. This can be explained by the model, which shows that the electron ion collisions are the dominating process. Further sensitivity studies have been performed. The EBW current rises linearly with the driving power. The dependence on the additional non-modulated power is week, but shows an increase of the current of $20 \%$ when the power is varied from $3 \mathrm{~kW}$ to $6 \mathrm{~kW}$. Probably, here the higher number of supra-thermal electrons is compensated by the unavoidable density increase with the total power. The Fish-Boozer-mechanism is 
assumed to be responsible for the EBW driven current. The Okhawa mechanism can be excluded since for large $N_{\|}$the interaction in the phase space takes place far from the trapped particle region. The EBW-current was calculated from the formula in [3]. The knowledge of the total EBW driven current is probably not sufficient to test the modelling, but the low temperature plasma gives access to measurement of the current density directly by current probes, which are moved through the plasma during the discharge. These mini-Rogowski coils consist of a stainless steel tube of $1.3 \mathrm{~mm}$ diameter, which forms a loop of $10 \mathrm{~mm}$ diameter. Inside the tube there is a coil with typically 500 windings. The tube is covered by insulating ceramics. The measured current density profile exhibited two counteracting currents at a magnetic field of $0.65 \cdot B_{c}$ as shown in Fig. 5. It should be noted that most of the current is driven at the outer radii, thus the total current is negative.
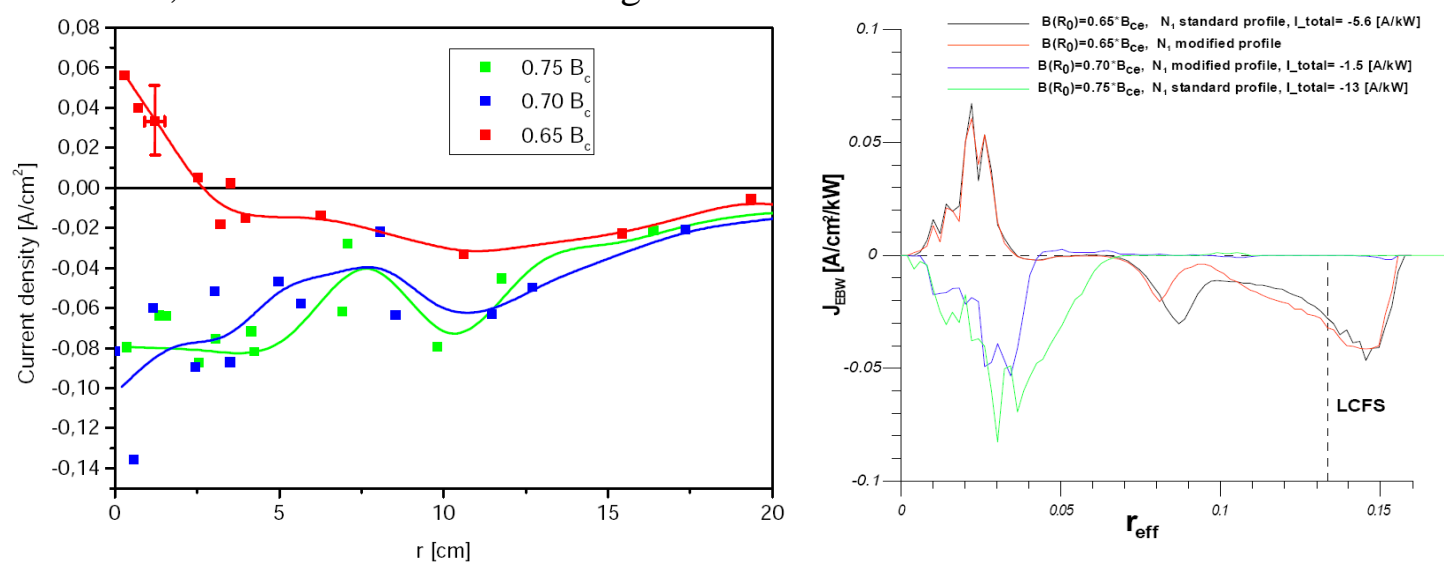

FIGURE 5. left) Experimental current density profiles measured by a movable Rogowski coil for 6 $\mathrm{kW}$ injected power. right) Calculated current density for different central toroidal field.

But the characteristic's positive current contribution could also be predicted by the model. Even more the positive central current vanishes when the magnetic field is increased, which is consistent with the model prediction. The total current of 7.5-15 $\mathrm{A} / \mathrm{kW}$, measured by the Ohmic transformer is comparable with the code prediction of 5.6-13 A/kW. However, the uncertainty of the electron distribution prohibits a more precise prediction.

\section{EXPERIMENTS WITH 28 GHZ EBWS}

The main challenges in achieving an EBW heated plasma is to find the optimal launch angle and to overcome the density threshold for OXB-mode conversion, which is $1 \cdot 10^{19} \mathrm{~m}^{-3}$. The first is straight forward in a current-less stellarator, since the magnetic configuration is mainly determined by external coil currents. The density threshold can hardly be overcome with $28 \mathrm{GHz}$ only, since the cut-off density of the best absorbed X2-mode is $0.5 \cdot 10^{19} \mathrm{~m}^{-3}$. In addition for best OXB-conversion the microwaves should be obliquely launched with $\mathrm{O}$-mode polarisation (left hand side elliptically polarized). Therefore, a target plasma must be generated by an additional heating scheme with the available $2.45 \mathrm{GHz}$. Even though no resonance condition is fulfilled at $0.5 \mathrm{~T}$, resistive absorption should generate plasma density. The $2.45 \mathrm{GHz}$ 
waves are launched with a double slot antenna, which generates a large $N_{\|}$number (>0.7) for the $2.45 \mathrm{GHz}$ waves. Due to multiple reflections at the metallic vacuum vessel the waves can couple with R-waves (whistler waves), which have no density limit for propagation. Resistive absorption generates a high density but low temperature plasma, which is appropriate to be taken over by EBW heating. The threshold density was reached with typically $20 \mathrm{~kW}$ magnetron power as shown in Fig 6. At that point the EBW-heating became effective. The power was absorbed at the plasma centre and the density was increased and became strongly peaked. After switching off the $2.45 \mathrm{GHz}$ power a purely EBW heated plasma was sustained. The sniffer diagnostic showed a drop down to $20 \%$ of the power level with no ECRH absorption. The radiation profile is strongly peaked in that case as shown in Fig. 7. The total radiated power is above $50 \%$ of the heating power. Therefore, the achievable density is limited by the ECRH power. The plasma temperature should be detected by the EBE-diagnostics, but when the transition into the OXB-heated plasma took place, a radiation temperature of more than $10 \mathrm{keV}$ was measured in the central channels.

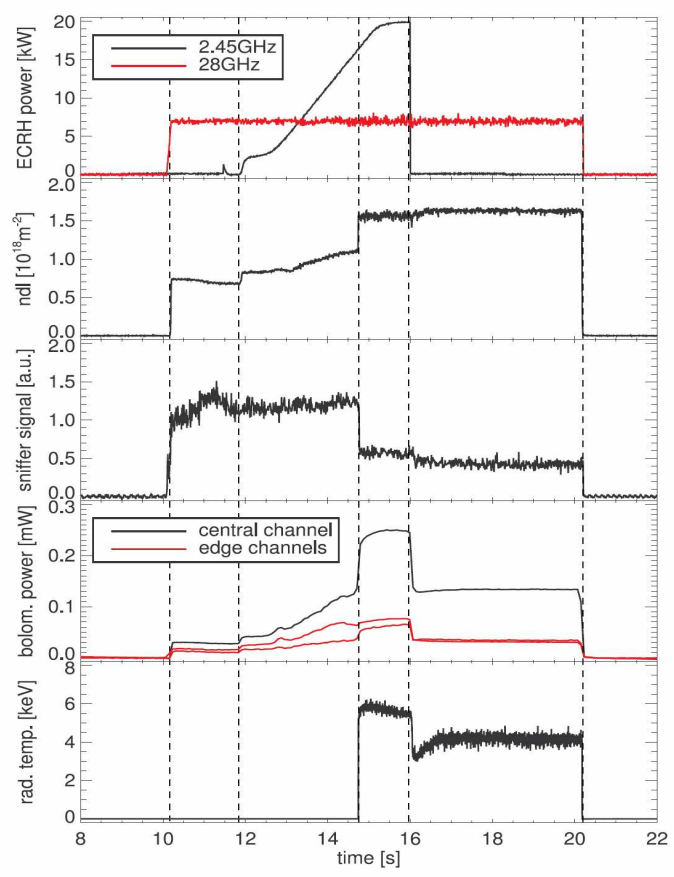

FIGURE 6. Time traces of an OXB-heated plasma operation. The transition takes place at $14.8 \mathrm{~s}$.

The EBE-spectrum was strongly peaked around the $28 \mathrm{GHz}$ resonance with peak levels of $20 \mathrm{keV}$. The radiation temperature is decreasing with the collision cross section and pressure of the operating gas (helium and argon). This intensive radiation originated from fast electrons, which are generated by the EC-interaction. This could be confirmed by the X-spectrum which also shows electron energies above $10 \mathrm{keV}$ in the OXB-phase exclusively. The highest BXO emission frequency is detected for 33 $\mathrm{GHz}$, thus the peak density must have been above $1.4 \cdot 10^{19} \mathrm{~m}^{-3}$. The resonant absorption was confirmed by a magnetic field scan. Best central deposition was found between 0.46 and $0.48 \mathrm{~T}$, which confirmed the low Doppler shift as predicted by raytracing calculations. 


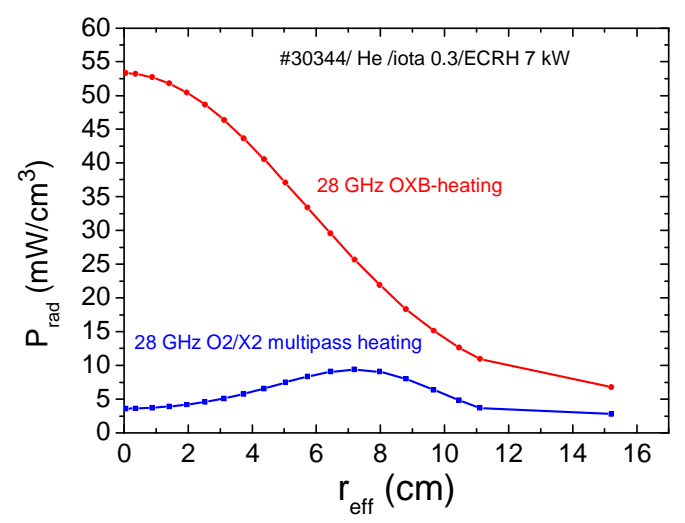

FIGURE 7. Radiation profile of over-dense EBW-heated plasma with central total single pass absorption in comparison with the profile of a low density plasma with multi-pass $\mathrm{O} 2$ heating. The profiles are reconstructed from the signals of a 12-channel bolometer camera.

\section{SUMMARY AND CONCLUSIONS}

At a low magnetic field (55 mT) a highly overdense plasma $\left(1 \cdot 10^{18} \mathrm{~m}^{-3}\right)$ could be achieved by EBW-heating with $2.45 \mathrm{GHz}$. The EBW were exited by OXB-mode conversion, which could be investigated in detail by RF-probes in comparison with full wave calculations. The EBW propagation was calculated with a 3 dimensional ray-tracing code which reproduces the measured Doppler down shifted absorption. It also reproduces the EBW driven current density, which was measured by small Rogowski coils inside the low temperature (10-20 eV) plasma. Thus these experiments have been clearly emerged as good benchmarks of modeling EBW-heating and current drive.

Steady state fully $28 \mathrm{GHz}$ EBW-heated plasma operation was achieved at the second harmonic resonant field of $0.5 \mathrm{~T}$ and densities above $1.4 \cdot 10^{19} \mathrm{~m}^{-3}$. The EBEdiagnostics measured an extremely high radiation temperature, which origin from a fast supra-thermal electron population generated by the EC-absorption of EBWs. Note, that in contrast to the electromagnetic waves the EC-interaction of EBWs is not limited in energy. This is a new unexplored plasma regime, which can give access to new wave particle interaction schemes as well as to advanced current drive scenarios with EBWs.

\section{REFERENCES}

1. Preinhaelter J., and Kopecký V., J. Plas. Phys. 10, 1 (1973).

2. Y.Y. Podoba, H. P. Laqua, G. B. Warr, M. Schubert, M. Otte, S. Marsen, F. Wagner and

E. Holzhauer, Phys. Rev. Lett. 98, 255003 (2007)

3. F.R. Hansen et al., Plasma Physics and Contr. Fusion, 27, (1985) 1077

4. H.P. Laqua et al., Phys. Rev. Lett. 78, 3467-3470 (1997).

5. A. Mueck et al., Phys. Rev. Lett. 98, 175004 (2007)

6. Y.Yoshimura et al., Plasma and Fusion Research: Rapid Communications Volume 1, 029 (2006)

7. H.P. Laqua et al., Phys. Rev. Lett. 90, 075003 (2003).

8. J. Urban and J. Preinhaelter, Journal of Plasma Physics, 72 (2006), 1041-1044

9. M. Otte et al., AIP Conf. Proc., Vol. 993, pp. 3-10 